\title{
Meal context and food preferences in cancer patients: results from a French self-report survey
}

\author{
Estelle Guerdoux-Ninot 1,2*, Robert D. Kilgour ${ }^{3,4}$, Chloé Janiszewski ${ }^{5,6}$, Marta Jarlier ${ }^{7}$, Jocelyne Meuric ${ }^{8,11}$, \\ Brigitte Poirée ${ }^{9,11}$, Solange Buzzo ${ }^{10,11}$, Grégory Ninot ${ }^{1,2}$, Julie Courraud ${ }^{1}$, Wendy Wismer ${ }^{12}$, Simon Thezenas ${ }^{7}$ \\ and Pierre Senesse $e^{1,2,5,11}$
}

\begin{abstract}
Purpose: The present study examined patient self-reports of descriptions, experiences and consequences of meal disturbances and food preferences within a cultural context (i.e., French meal traditions) in various treated cancer patients along their disease trajectory.

Methods: Over 800 questionnaires were sent to 20 cancer treatment centres in France. During a 9-month period, 255 questionnaires were received from five centres. Inclusion criteria included those French patients over 18 years of age, could read and understand French, had an Eastern Cooperative Oncology Group score between 0 and 2, experienced treatment-induced nutrition changes and/or had decreased oral intake. Dietetic staff assessed clinical characteristics while patients completed a 17-item questionnaire.

Results: The majority of patients were diagnosed with breast, gastro-intestinal (Gl) tract and head and neck cancers (62\%). Half of the patients ( $49 \%$ ) experienced weight loss $>5 \%$. The main treatment-induced side effects were fatigue, nausea, dry mouth, hypersensitivity to odors and GI tract transit disorders. These discomforts affected eating and drinking in $83 \%$ of patients, inducing appetite loss and selected food aversion. Food preference appeared heterogeneous. Food taste, odor and finally appearance stimulated appetite. Finally, dietary behaviors and satisfaction were driven by the extent to which food was enjoyed.

Conclusions: During oncologic treatments, eating and drinking were affected in more than three-quarters of patients. As recommended by practice guidelines, nutritional assessment and follow-up are required. Personalized nutritional counseling should include the role of the family, patient's meal traditions, and food habits.
\end{abstract}

Keywords: Cancer, Food preferences, Taste, Meal context, Feeding behaviors, Weight loss

\section{Background}

In disease-free adults, daily food intake is distributed over many meals and snacks. The traditional meal definition in the European culture is a social event that occurs on a regular daily basis at relatively fixed hours, including several dishes presented in succession (Bellisle et al.

\footnotetext{
*Correspondence: estelleg.ninot@yahoo.fr;

estelle.guerdoux-ninot@icm.unicancer.fr

${ }^{1}$ SIRIC Montpellier Cancer, Cancer Institute of Montpellier (ICM)Val d'Aurelle, 208 avenue des Apothicaires, Parc Euromédecine, 34298 Montpellier Cedex 5, France

Full list of author information is available at the end of the article
}

2003). Traditionally, French adults are used to ingesting three meals a day. The interval that separates lunch from dinner in the French culture is traditionally interrupted by a small snack that produces long lasting satiety and that accounts for, on average, $18.5 \%$ of the total daily energy intake (Bellisle et al. 2003). However, several factors affect meals in the natural environment, such as social facilitation, subjective hunger, food hedonics, learned habits, and palatability. Food hedonics is one component of the eating and drinking experience and refers to a psychological determination of the extent to which eating and drinking provides feeling of pleasure or 
displeasure (Boltong et al. 2012). Palatability is defined as the stimulus quality of a substance which determines its acceptability (de Castro et al. 2000). In a study including French participants, higher levels of palatability were found to be related to larger meal portions, meal duration, smaller satiety ratios, greater hunger, and less anxiety/depression (de Castro et al. 2000). Incidentally, palatability has large effects on intake regardless of culture (i.e., for the North American and the French cultures) but appears to be only one of many factors influencing intake (de Castro 2000).

Moreover, several studies have demonstrated an altered food preference in cancer, and food-related quality of life depending on treatment or disease stage (Epstein et al. 2002; Hovan et al. 2010). Patients with head and neck cancers had altered food-related expectations and changes in the "meaning of food" resulting in physical, emotional and social losses (McQuestion et al. 2011). Following curative gastrectomy, patients experienced increased dysphagia, eating restriction, anxiety, taste changes, and body image scores which gradually decreased over 12 months (Kong et al. 2012). Radiotherapy is also well known to impair taste perception during and after cancer treatment (e.g., Ruo Redda and Allis 2006; Irune et al. 2014). Taste changes and food preferences are also expressed differently according to chemotherapy (CT) regimen with significant relationships between ageusia and dry mouth, bitter taste and appetite loss, sour taste and nausea, and anorexia and dry mouth (Zabernigg et al. 2010). As highlighted by recent systematic reviews, the influence of CT changes in food preferences have not been consistently demonstrated (Epstein and Barasch 2010; Boltong and Keast 2012; Gamper et al. 2012). Finally, the extent to which these disturbances play a role in dietary behavior during and after treatment remains relatively unknown. Despite randomized trials of dietary counseling compared to no dietary counseling or to standard practice (e.g., Isenring et al. 2007), there is a lack of information concerning practical management of food intake, particularly meal and food preferences in a cultural context among cancer patients undergoing treatment.

The approach of this study was to explore meal and food preferences among cancer patients within a cultural context (i.e., including meal traditions) because of its pregnant role in feeding behaviors. Therefore, we conducted a multi-centre study to investigate self-reported descriptions and experiences of the meals context and food preferences in cancer patients.

\section{Methods}

\section{Study population and design}

Over a 9-month period, patient self-report surveys were completed anonymously and used to explore patient descriptions, experiences and consequences of meal disturbances.

Height hundred questionnaires were sent from the lead investigation site (Cancer Institute of Montpellier, ICM) to 20 cancer treatment centres in France. Ethical approval to conduct this study and to publish the results was granted from the government through the "Oséo program" Grant. Patients were eligible for inclusion if: (1) they were aged 18 years or over; (2) they were French by culture, were able to read and understand French; (3) they had an Eastern Cooperative Oncology Group (ECOG) Performance Status score of 0, 1 or 2; (4) they had self-reported oral intake changes related to treatment during the dietician clinical assessments; and/or 5) they had an ingesta visual analogue scale (ingesta-VAS) score $<8$ (Thibault et al. 2011; Senesse et al. 2014). The ingestaVAS was used for a quick assessment of dietary intake in clinical practice, particularly in patients with weight loss $(0=$ no ingesta; $10=$ usual ingesta). This scale is highly correlated with caloric intake $(p<0.0001)$. Patients defined themselves their level of ingestion of food by ticking a $100-\mathrm{mm}$ line traced on a paper to answer the inquiry "How much do you currently eat on a scale from 0 "nothing at all" (far left side of the line) to 10 "as usual" (far right side of the line)?" (Thibault et al. 2011).

\section{Material and procedure}

In line with Boltong et al. (2012), the questionnaire was developed by a working group of students, dietitians, medical residents, physicians, and food industry professionals with product development experience. A pilot test of the questionnaire was conducted with 20 patients to ensure item clarity. No change was made to the questionnaire following this test phase.

Dietitians assessed patient demographic and clinical characteristics (e.g., tumor location, anticancer treatment, weight loss), and provided instruction for completion of the questionnaire. The questionnaire consisted of 17 items (Table 1) that related to the description of (1) treatmentrelated side effects and their impact on eating and drinking; (2) cooking skills; (3) dietary behavior; (4) food preference; (5); experiences with nutrient-enriched food; and (6) preferences for a new dietary product. The questionnaire contained open-ended and multiple-choice questions with an opportunity to provide alternative answers and to choose more than one answer. Two additional questions were about food attributes that stimulate appetite (\#4.4) and the advantages that motivate the purchase of new dietary products (\#6.1). For these two items, the answers were ranked in descending order of importance on a 7-point and a 9-point scale, respectively $(1=$ most important $)$.

Patients' written informed consent was obtained prior to the completion of the questionnaire. Institutional 


\section{Table 1 Self-report questionnaire ${ }^{a}$}

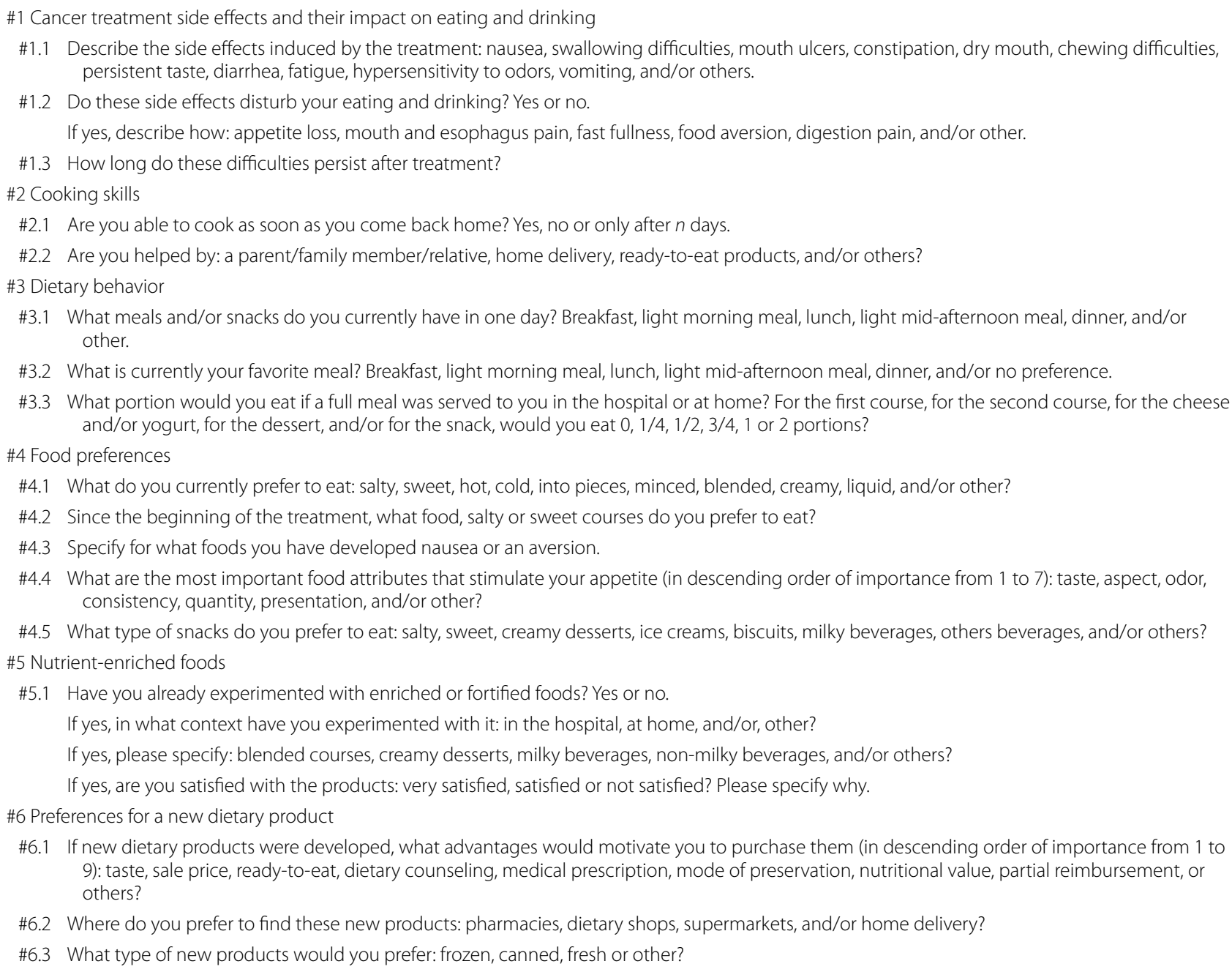

${ }^{a}$ Translated from French to English

review boards of each investigational site approved the study protocol. Questionnaires were returned to the ICM for data analysis.

\section{Statistical considerations}

Categorical variables were reported by means of frequency and percentages. For continuous variables, medians, means, standard deviations and range values were computed. The association between demographic, clinical characteristics and different items included in the self-report questionnaire was assessed using Pearson's Chi square test or Fisher's exact test when applicable for categorical variables, and using Kruskal-Wallis or Student $T$ test for continuous variables.

All reported p-values are two-sided and were considered significant at the $5 \%$ level. Data from open-ended questions were handled and analysed with NVivo10 software, which usually supports qualitative methods research. In the current study, they were explored using descriptive content analysis. Themes are only reported with quotes considered to be typical unless explicitly noted (Sandelowski 2010). Only brief comments from the qualitative analysis are reported. Statistical analysis was performed using STATA v.11.0 software (Stata Corporation, College Station, TX, USA).

\section{Results}

Patient sample

From the 800 distributed questionnaires, 255 were returned (31.8\%) from the 20 oncology-specialized hospitals, in particular from the ICM, the Francois Baclesse Center of Caen, the Curie Institute of Paris, the Paoli Calmettes Institute 
of Marseille, and the Antoine Lacassagne Center of Nice. Patients completed the questionnaires as outpatients (52.1\%), or inpatients (full-time hospitalization, $47.8 \%$ ). Patient characteristics are shown in Table 2. Patients were on average 59.5 years old ( \pm 13 years, SD), ranging from 22 to 89 years. All of them were French by culture and adopted French meal traditions. Most were women (60.0 \%), aged from 50 to 69 years (56.4\%), lived as a couple (69.8\%), had one or two children $(64.4 \%)$ and did not work (48.2\%) or were on sick leave (30.2\%). Tumors were localized mostly in breast (27.1\%), GI tract (22.0 \%) and head and neck (12.9\%). Most patients received a single round of CT (71.4\%). Most patients had altered functional abilities; $39.2 \%$ of patients were restricted in their ability to conduct physically strenuous activities but were ambulatory and able to carry out work of a light or sedentary nature (ECOG performance status score of 1 ), and $28.2 \%$ were ambulatory and capable of all self-care but unable to carry out any work activities (ECOG score of 2). Half of patients (50.4\%) suffered from weight loss $\geq 5 \%$ compared with their pre-treatment weight (Fearon et al. 2011). Insufficient caloric intake, as defined by a score $<8$ at the ingesta visual analogue scale, was reported in $54.1 \%$ of cases. Dietitians noticed that twothirds of patients suffering from weight loss $\geq 5 \%$ (65.7 \%) consumed sip feeds, $48.0 \%$ split meals and $15.8 \%$ had artificial nutrition. Side effects of cancer treatment impacting oral intake are detailed in Table 3.

\section{Responses to the themes of the self-report questionnaire \#1 Treatment-related side effects and impact on eating and drinking}

The main side effects induced by the treatment therapies were fatigue $(74.5 \%)$, nausea $(55.3 \%)$, dry mouth (48.6\%), hypersensitivity to odors (35.7\%) and constipation (34.1\%). Most patients (54.5\%) reported digestive tract disorders such as constipation or diarrhea (Table 3). Treatment-related side effects affected eating and drinking in $83.1 \%$ of patients, principally inducing appetite loss (66.8\% of patients who reported an impact on eating and drinking), food aversion (51.4\%) and satiety (39.7 \%) (Table 4). These disturbances on eating and drinking persisted for 10.8 days on average after treatment (ranging from 1 to 120 days). From open questions in the questionnaires, we also identified patient-related theme of feeling disturbed by the side effects. The strongest message from patients was that they experienced taste modifications such as "I have no taste anymore" (centers of Marseille and Caen), "I have lost taste" (center of Montpellier), "my taste has been modified" (center of Nice), "I have strange taste in my mouth" (center of Paris). They also qualitatively reported a loss of pleasure described as
Table 2 Patient demographic and clinical characteristics $(n=255)$

\begin{tabular}{|c|c|}
\hline Characteristic & $n(\%)$ \\
\hline \multicolumn{2}{|l|}{ Age } \\
\hline$<60$ years & $111(43.5)$ \\
\hline$\geq 60$ years & $144(56.5)$ \\
\hline Female sex & $153(60.0)$ \\
\hline \multicolumn{2}{|l|}{ ECOG performance status ${ }^{\mathrm{a}}$} \\
\hline 0 & $76(29.8)$ \\
\hline 1 & $100(39.2)$ \\
\hline 2 & $72(28.2)$ \\
\hline Side effects on oral intake & $244(95.7)$ \\
\hline \multicolumn{2}{|l|}{ Ingesta visual analogue scale ${ }^{b}$} \\
\hline$(0-6)$ & $90(35.3)$ \\
\hline (7 and 8) & $48(18.8)$ \\
\hline$(8-10)$ & $100(39.2)$ \\
\hline Artificial nutrition & $38(15.8)$ \\
\hline \multicolumn{2}{|l|}{ Personal and professional status } \\
\hline Live in southern France & $143(56.1)$ \\
\hline As a couple & $178(69.8)$ \\
\hline Active $^{c}$ & $27(10.6)$ \\
\hline Inactive $^{d}$ & $123(48.2)$ \\
\hline Sick leave & $77(30.2)$ \\
\hline \multicolumn{2}{|l|}{ Tumor location } \\
\hline Breast & $69(27.1)$ \\
\hline Digestive system & $56(22.0)$ \\
\hline Head and neck & $33(12.9)$ \\
\hline Hematopoietic system & $18(7.1)$ \\
\hline Lung & $17(6.7)$ \\
\hline Gynecology & $17(6.7)$ \\
\hline Others & $35(13.7)$ \\
\hline \multicolumn{2}{|l|}{ Anticancer treatment } \\
\hline Chemotherapy & $182(71.4)$ \\
\hline Radiotherapy & $7(2.7)$ \\
\hline Chemotherapy + radiotherapy & $26(10.2)$ \\
\hline Surgery & $23(9.0)$ \\
\hline Others (e.g., antibiotic therapy) & $11(4.3)$ \\
\hline \multicolumn{2}{|c|}{ Weight loss (compared with baseline) (\%) } \\
\hline$<5$ & $121(47.5)$ \\
\hline $5-10$ & $59(23.1)$ \\
\hline $10-20$ & $53(20.8)$ \\
\hline$\geq 20$ & $13(5.1)$ \\
\hline
\end{tabular}

a The Eastern Cooperative Oncology Group (ECOG) performance status refers to functional ability scores (ranged from 0 to 5 ) to quantify cancer patients general well-being and activities of daily life

b The ingesta visual/verbal analogue scale is used for a quick assessment of dietary intake in clinical practice, particularly in patients with weight loss $(0=$ no ingesta; $10=$ usual ingesta). This scale is highly correlated with caloric intake. Patients defined themselves their level of ingestion of food between 0 (nothing) and 10 (as usual)

c Patients were considered to be active if they had a job

d Patients were considered to be inactive if they had no job or were retired 
Table 3 Treatment side effects

\begin{tabular}{lc}
\hline Type of side effects $(\boldsymbol{n}=\mathbf{2 5 5})$ & $\boldsymbol{n}(\mathbf{\%})$ \\
\hline Fatigue & $190(74.5)$ \\
Nausea & $141(55.3)$ \\
Dry mouth & $124(48.6)$ \\
Hypersensitivity to odors & $91(35.7)$ \\
Constipation & $87(34.1)$ \\
Diarrhea & $83(32.5)$ \\
Mouth ulcers & $64(25.1)$ \\
Swallowing difficulties & $62(24.3)$ \\
Vomiting & $55(21.6)$ \\
Persistent taste & $36(14.1)$ \\
Chewing difficulties & $35(13.7)$ \\
Others (e.g., dysgeusia) & $52(20.4)$ \\
\hline
\end{tabular}

Table 4 Side effects and oral intake impact

\begin{tabular}{lr}
\hline Type of perturbation of oral intake $(\boldsymbol{n}=\mathbf{2 1 2})$ & $\boldsymbol{n}(\mathbf{\%})$ \\
\hline Appetite loss & $143(66.8)$ \\
Food aversion & $110(51.4)$ \\
Satiety & $85(39.7)$ \\
Mouth pain & $54(25.2)$ \\
Abdominal pain during digestion & $26(12.1)$ \\
Others (e.g., ageusia) & $23(10.7)$ \\
\hline
\end{tabular}

"everything considerate, I feel a discomfort" or explicitly as "I have lost pleasure when eating and drinking".

\section{\#2 Cooking skills}

Over half the patients (53.3\%) were able to cook as soon as they returned home from a hospital day or from a treatment session (i.e., after a hospital stay), and $25.9 \%$ started cooking again after 4-5 days. Patients' parents were the main source of help $(84.7 \%)$ irrespective of the patients' familial situation (i.e., as a couple or not).

\section{\#3 Dietary behavior}

Patients reported to have daily dinner (91.8\%), breakfast $(90.2 \%)$ and lunch $(88.2 \%)$. Outpatients (47.9\% of patients) had more lunch meals than inpatients (91.8 vs. $83.0 \%, p=0.042)$ and tended to have more dinners (95.1 vs. $88.4 \%, p=0.061)$. However, breakfast was identified as the favorite meal for $55.7 \%$ of patients when compared with lunch and dinner ( $29.8 \%$ and $20.4 \%$, respectively), whether they were an inpatient or outpatient.

A typical French meal consists of 4 courses: typically the "first course" (e.g., salad) is followed by the "second course" (e.g., meat with vegetables), then completed by some "cheese and/or yogurt", and finally by a "dessert" (e.g., fruit or cake). Regarding the portion that patients would eat if a full French meal was served in the hospital or at home, $29.4 \%$ declared that they did not eat a first course. Conversely, $36.5 \%$ reported they would eat one portion of the second course and $23.9 \%$ would eat half a portion. Most patients (58.4\%) would eat one portion of cheese and/or yogurt, and $56.1 \%$ thought they would eat one dessert portion. Similarly, a typical French snack (around 11 a.m. or 4 p.m.) consists of fruit or cake with a tea or coffee. Over half $(57.3 \%)$ the patients declined to snack.

Furthermore, results showed a significant difference between the type of hospitalization and the consumption of the first and second courses. When compared with inpatients, a significantly higher percentage of outpatients declared to eat one entire portion or more of the first courses (33.3 vs. $20.0 \%, p=0.024)$ and second courses ( 47.9 vs. $24.6 \%, p<0.001)$.

Finally, data from open-ended question were mainly linked to a reduction of the usual eaten portions. A patient explained for example that "during five days, I [he] don't eat afternoon snacks anymore... and I [he] don't eat much..." while another reported that "I [he] don't eat anymore during the chemotherapy" or "I must eat very light because all that is solid hurts my esophagus".

\section{\#4 Food preferences}

Most patients preferred salty foods (57.6\%) rather than sweet ones $(39.6 \%, p<0.001)$ in general. They reported a preference for hot $(58.7 \%)$ rather than cold food (29.8\%), $p<0.001)$. Concerning the textures, food cut into pieces was preferred by $27.8 \%$ of patients, followed by creamy (20.8\%), liquid (14.1\%), minced (13.7\%) and lastly by blended food (9.8\%). However, sweet foods were as valued as salty foods when patients detailed their preferences. Qualitatively, the strongest message from patients was that the seasoning level drove their preferences, for example "I prefer foods that are spicy", "foods that are peppery" or conversely "I eat strictly without salt" or "I like dishes that are not too much salty nor too much sweet". Results about the descriptive preferences are then reported as percentages of responses in Table 5. The most enjoyed foods were fruits ( $49 \%$, including compotes), dairy products (45\%), and after, pasta, and red meat $(40 \%)$. On the other hand, more than two-thirds of patients (69\%) reported an aversion to specific foods, particularly red meat $(15.4 \%)$ and meals with sauce (10.9\%). The key theme identified was the preference for the typical French courses. It included French specialties (e.g., "quiche", "bread") and also the Mediterranean diet, incorporating a majority of "fruits and vegetables", "fish" and "soup".

Patients then ranked a list of the most important attributes stimulating appetite (i.e., taste, appearance, odor, 
Table 5 Food preferences and aversions in cancer patients

\begin{tabular}{lc}
\hline & $\boldsymbol{n}(\%)$ \\
\hline Main preferences $(n=205)$ & $100(48.8)$ \\
Fruits & $92(44.9)$ \\
Dairy products & $86(42.0)$ \\
Pasta & $82(40.0)$ \\
Red meat & $74(36.1)$ \\
Mashed potatoes & $74(36.1)$ \\
Vegetables & $68(33.2)$ \\
Soup & $62(30.2)$ \\
Fish & $42(20.5)$ \\
White meat & $40(19.5)$ \\
Milky dessert & $26(12.7)$ \\
Rice & $20(9.8)$ \\
Salad & \\
Main food aversions $(n=175)$ & $27(15.4)$ \\
Red meat & $19(10.9)$ \\
Meals and meat with sauce & $13(7.4)$ \\
Everything & $9(5.1)$ \\
Sweet & $7(4.0)$ \\
Chocolate & $7(4.0)$ \\
Cheese & $7(4.0)$ \\
Coffee &
\end{tabular}

texture, quantity, packaging and other) in descending order of importance on a 7-point scale $(1=$ most important). Taste was the most frequently cited quality to stimulate appetite (mean score of 2.4), followed by odor and appearance (both 3.0), presentation (4.1), consistency (4.3) and finally quantity (4.6) (Fig. 1). Some patients added qualitatively that the temperature was as another important attribute that stimulated appetite.

Concerning snacks, less than half the patients did not report any taste (41.6\%) and snack type preference $(47.5 \%)$. However, patients indicating a taste preference preferred sweet snacks $(46.7 \%)$ rather than salty ones $(15.7 \%, p=0.003)$ with no significant difference among biscuits (32.5\%), creamy desserts (16.5\%) and ice cream $(20.4 \%, p=0.34)$. In summary, most of patients had reported a preference for salty meals but for sweet snacks. Interestingly, patients who lived in southern France (i.e., 56.1 \% lived in Nice, Marseille or Montpellier) had a greater preference for ice creams compared with patients living in the north (26.6 vs. $12.5 \%, p<0.05)$. No other difference was found in responses from the diverse geographical areas. Milky beverages $(17.6 \%)$ were preferred less than other beverages $(38.0 \%)$ such as tea, coffee or herbal tea (without stimulant) and fruit juices $(p=0.002)$. Finally, answers from open-ended question focused mostly on "fruits" as another potential snack.

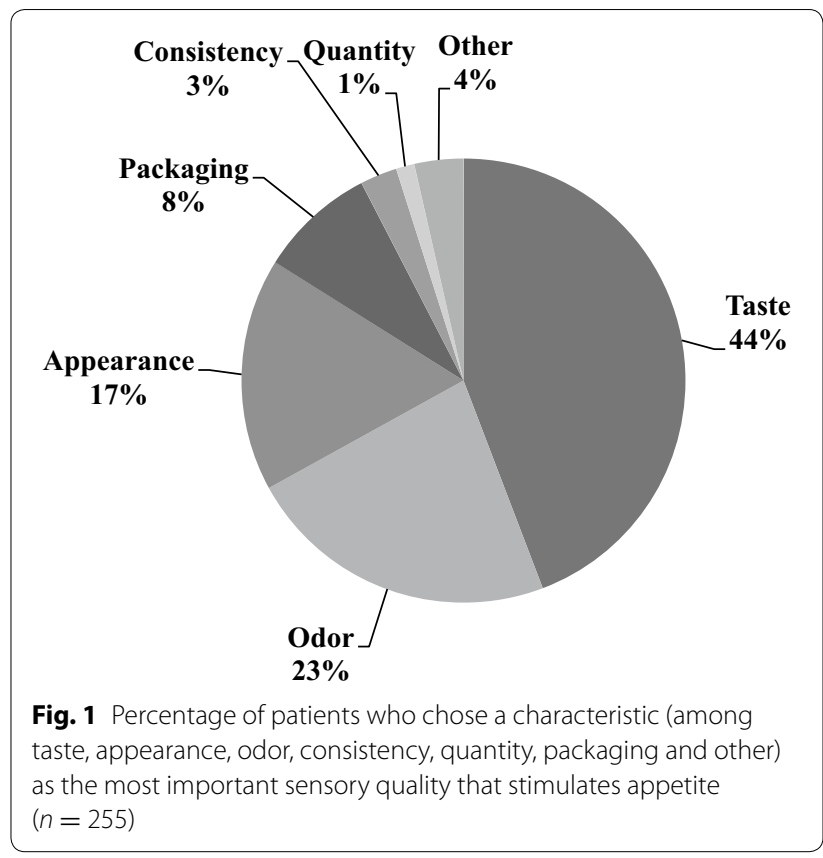

\section{\#5 Nutrient-enriched foods}

Fifty-four percent of patients $(n=129)$ had already experimented with enriched or fortified foods, $43.4 \%$ at home, $36.4 \%$ in the hospital, $19.4 \%$ at both places. Oral nutritional supplements were mostly milky beverages (69.8\%), creamy desserts (29.5\%), and non-milky drinks (24.0\%). From the open-ended question, most patients reported to have already eaten hyperprotein soup. More than two-thirds of patients $(70.5 \%)$ were satisfied or very satisfied with the products. Among those who expressed dissatisfaction with the nutrient-enriched foods, the majority (84\%) identified taste as the major cause of their dissatisfaction. Qualitative data enlighten that the products were mostly liked because they were "easy to digest" while they were mostly disliked because of their "bad, viscous or stodgy taste".

\section{\#6 Preferences for a new dietary product}

Of the characteristics that ranked the motivation of purchasing a new dietary product (from 1 to 9 , where $1=$ most important), taste was considered to be the most important (mean score of 2.4), followed by nutritional value, dietary counseling (both 3.8 ), and medical prescription (4.4). Patients expressed a desire to find these new products in supermarkets (59.6\%), community pharmacies $(29.0 \%)$ and dietary shops (20.4 \%). Home delivery would also be appreciated by $17.3 \%$ of patients. Preference for product form was for fresh (74.1\%), frozen $(25.9 \%)$ and canned products $(17.3 \%)$. The other themes that patients freely expressed were the visual aspect of 
the product (e.g., "presentation, color, appearance") and its "organic" character.

\section{Discussion}

This is one of the first studies to describe meal-related behaviors and food preferences of cancer patients within a cultural context (i.e., considering French meal traditions). We used patient self-reports to investigate descriptions and experiences of the meal situation focusing on symptoms, behaviors, and changes. The majority of patients had a good ECOG performance status (about $70 \%$ were grade 0 or 1 ), and more than $90 \%$ received an oncologic treatment. Indeed, the first remarkable result was that treatment-related side effects affected eating and drinking in $83.1 \%$ of cases irrespective of tumor location and of type of treatment.

In fact, the main consequence of altered eating and drinking was decreased food and energy intake. In our study, more than $50 \%$ of patients suffered weight loss $\geq 5 \%$, increasing their risk of potential health complications (Dewys et al. 1980; Andreyev et al. 1998; Bellisle 2003; Argilés 2005; Arends et al. 2006; Senesse et al. 2014). Most patients had breast or GI tract cancer treated with chemotherapy. Indeed, the main treatment-induced side effects included fatigue, nausea, dry mouth, altered sensations to smell and digestive transit disorders. In line with previous studies (Zabernigg et al. 2010; Coa et al. 2015), these side effects affected eating and drinking in $83 \%$ of patients, by inducing principally appetite loss and aversion to specific foods. Due to the variety of symptoms affecting patients, it is essential to provide a comprehensive approach integrating dietary advice and other specialists such as speech language pathologists, occupational therapists or social workers, depending on the disease and treatment (Senesse et al. 2014). Further studies showed that dietary counseling reduced weight loss, improved quality of life, and reduced toxicity in irradiated patients with head and neck, and gastrointestinal cancer (Ravasco et al. 2005a, b; Isenring et al. 2012). Dietary counseling involving the prescription of a therapeutically adjusted diet should be more widely provided to such patients. Nutritional guidelines highlight the importance of dietary counseling; however, there is a lack information concerning the definition of dietary counseling used to demonstrate its benefits (Boltong et al. 2012).

Dietary counseling must include meal and food preferences within the cultural context and the patient's home situation. Three out of 4 patients were concerned with fatigue and were not able to resume cooking and meal preparation immediately after treatment. Patients' parents were the main source of help even if the patient was as a couple. Hence, dietary counseling should also involve them, and more broadly the family. Then, cancer-related fatigue is well known to be one of the most prevalent and burdensome symptoms (Servaes et al. 2002; Tomlinson et al. 2013) and often becomes a major distressing symptom (Minton et al. 2013). The development of a specific physical activity intervention could reduce asthenia and fatigue, and may stimulate appetite thus leading to an increase in oral intake (Bortolon et al. 2014). Concerning meal-related behaviors and cultural context, more than $50 \%$ of patients identified breakfast as their favorite meal. Patients also reported a preference for lunch and dinner at home rather than in the hospital, eating salty and hot meals, and food cut into pieces. In fact, our results highlight the importance of meals and hedonics food (i.e., the extent to which eating and drinking provides affect of pleasure or displeasure) for French oncologic patients, also documented among healthy adults in France (de Castro et al. 2000; Bellisle et al. 2003). In this study, meals (i.e., breakfast, lunch and dinner) provided the greatest contribution to food intake, while also demonstrating a specific role for snacks. The consumption of snacks would appear to be a simple way to increase energy and protein intake. For example, almost half of all patients ate at least one portion of cheese and/or yogurt, and also one dessert portion with ice cream preference in the southern region of the country.

Oncologic treatment effects may differentially affect food preference as taste quality changes have been shown to be individual (Brisbois et al. 2011). Food preference information compiled from six studies indicates that caffeinated foods and drinks, red meat and citrus fruit or juices are more likely to become an aversion during CT than other foods or beverages (Boltong and Keast 2012). In a recent study among hospitalised haematological cancer patients, fresh fruit, ice cream, cheese and mashed potatoes with bacon were the most desired food items (Okkels et al. 2016). Our results were in agreement with a recent qualitative study in which patients expressed a reduced intensity of elements of flavor (taste "fading" or "blending out") (Coa et al. 2015; Okkels et al. 2016). Food hedonics are broader than the eating and drinking experience (Boltong and Keast 2012). They may include a good palatability and a psychological determination of the extent to which diet provides emotion of pleasure or displeasure. For example, in our sample, some patients reported enjoyment of fruit, dairy products and red meat, whereas these same foods repulsed others. Finally, qualitative data enlighten the importance of cultural habits: beyond the treatment-induced side effects, patients reported preferences for the typical French courses, in particular for the "Mediterranean diet".

Participants indicated that taste was the most important attribute stimulating appetite, and also the most important benefit rewarding the purchase of a new dietary 
product after the nutritional value itself. Also, odor and appearance seemed to influence appetite more than the quantity itself. Interestedly, a new cognitive propriety that may also influence appetite has emerged from the qualitative data: whether the food was organic or not. First, our results confirmed that there are many factors that affect meals in cancer context, as it was shown in the natural environment (de Castro et al. 2000). Palatability had only a limited influence on intake. Then, it is important to note that taste is often confused with flavors (Spence 2013). Taste perception refers to those sensations that are elicited by the stimulation of the gustatory receptors on the tongue (sweet, sour, salty, bitter and more recently, umami-savory) (Stevenson 2009). However, people virtually never experience pure isolated taste and mostly experience flavors, resulting from the combination of taste, retronasal olfaction and trigeminal inputs (Small 2012). "Fruity", "meaty", "floral" and "burnt" are all flavor descriptions (Verhagen 2007). Actually, flavor is perhaps the most multimodal of all of our sensory experiences (Auvray and Spence 2008). Therefore, it is now timely to suggest that cognitive neuroscientists integrate the study of multisensory flavor perception in the oncology field. As it was demonstrated with visual-auditory stimuli in older adults (Laurienti et al. 2006), the use of multiple sensory channels may represent an effective compensatory strategy to overcome the unisensory deficits induced by cancer treatment. There is hope that our growing psychological scientific understanding of multisensory flavor may help the food industries to deliver adjusted and more personalized products for better health care.

In summary, given their impact on morbidity, mortality and quality of life (Zabernigg et al. 2010), taste and/or smell changes and their impact on food hedonics should be considered very seriously in clinical practice. Such data should be integrated in tools assessing quality of life and should be used to improve nutritional status (Hutton et al. 2007).

Finally, we identified essential and practical advice for health-care professionals in order to improve energy intake and to prevent significant weight loss before and during treatment therapy.

- Eating and drinking are affected in most of cases during treatment. A systematic nutritional assessment is required as well as a follow-up as recommended in guidelines (Arends et al. 2006; August et al. 2009; Senesse et al. 2014).

- Focusing dietary counseling on meals, especially on breakfast, should be efficient to increase dietary intake addressed to in- and outpatients.

- At home, dietary counseling should also involve patients' parents who are the main source of help, and more broadly should involve the family. Therefore, lunch and dinner could be improved easily.

- Prescription of a therapeutic diet should be personalized and adjusted to the patients' food preferences and meal-related behaviors, including patient's culture (in terms of meal traditions), geographical area, and habits.

Additional investigations, including both qualitative and quantitative methods (i.e., mixed approaches), are necessary to further reduce the gap between self-report experiences and evidence-based strategies.

We have identified several limitations to our study. The questionnaire used to assess dietary changes may have been too long and complex for some patients who required the help of the oncology dietitian staff for its completion. Even if there were no biases with interpretation and responses by participants who required some help (it concerned only a few patients), it made heavy the procedure for those participants. Also, it did not clearly ask patients about the need for counseling and nutritional support, nor whether participants presently received this service. No information was given about the average length of time these patients have been receiving treatment. Our results should be interpreted and generalized with caution. These concerns relate specifically to the relatively low response rate and heterogeneity of the sample. Our study population was purposely heterogeneous, including patients with different types, locations, and stages of cancer, and also different therapies. While published studies usually focus on a precise disease and treatment, we aimed to reflect the diversity of patients met in real practice.

In conclusion, this study showed a high prevalence of symptoms reducing appetite and changes to meal and food preference during oncologic treatment. Individual dietary counseling has been shown to reduce morbidity and mortality, and to improve functional performance and well-being (Bauer 2007; Meuric and Besnard 2012). However international guidelines addressing dietary counseling should incorporate meal and food preferences in the cultural context specific to each country (i.e. meal traditions).

\footnotetext{
Abbreviations

Gl: gastro-intestinal; CT: chemotherapy; ECOG: Eastern Cooperative Oncology Group; ICM: Cancer Institute of Montpellier; ingesta-VAS: ingesta visual/verbal analogue scale.

\section{Authors' information}

The pilot EGN has been working as a psychologist and a neuropsychologist for more than 12 years in geriatrics, neurology and more recently in oncology. Her researches aim to understand the psychological, cognitive and dynamic processes involved in health-related quality of life and well-being. She wrote
} 
the manuscript and gave a human and social light in the data interpretation. Her contribution matched with the PS's points of view (the coordinator of Supportive Care and Head of Clinical Nutrition and Gastroenterology in the Cancer Institute of Montpellier) who conceived the study, and also completed the other authors' contribution of this study.

\section{Author details}

${ }^{1}$ SIRIC Montpellier Cancer, Cancer Institute of Montpellier (ICM)-Val d'Aurelle, 208 avenue des Apothicaires, Parc Euromédecine, 34298 Montpellier Cedex 5, France. ${ }^{2}$ Epsylon Research Unit EA 4556 Laboratory, Departments of Sport Sciences, Medicine and Psychology, University of Montpellier and University Paul Valery, Rue du Pr. Henri Serre, 34000 Montpellier, France. ${ }^{3}$ Department of Exercise Science, The Richard J. Renaud Science Complex, Room SP-165-17, Concordia University, Loyola Campus, 7141 Sherbrooke Street West, Montreal, QC H4B 1R6, Canada. ${ }^{4}$ McGill Nutrition and Performance Laboratory (MNUPAL), McGill University Health Centre, Suite 105B, Place Vendome, 5252 de Maisonneuve Ouest, Montreal, QC H4A 3S5, Canada. ${ }^{5}$ Department of Clinical Nutrition and Gastroenterology, Cancer Institute of Montpellier (ICM), Val d'Aurelle, 208 avenue des Apothicaires, Parc Euromédecine, 34298 Montpellier Cedex 5, France. ${ }^{6}$ Department of Clinical Research, Cancer Institute of Montpellier (ICM), Val d'Aurelle, 208 avenue des Apothicaires, Parc Euromédecine, 34298 Montpellier Cedex 5, France. ${ }^{7}$ Biostatistics Unit, Cancer Institute of Montpellier (ICM), Val d'Aurelle, 208 avenue des Apothicaires, Parc Euromédecine, 34298 Montpellier Cedex 5, France. ${ }^{8}$ Department of Dietetic and Nutrition, Curie Institute of Paris, 26 rue d'Ulm, 75248 Paris Cedex 05, France. ${ }^{9}$ Department of Dietetic, Centre Francois Baclesse of Caen, 3 avenue du Général Harris, BP5026, 14076 Caen, France. ${ }^{10}$ Department of Dietetic, Centre Antoine Lacassagne of Nice, 33 Avenue de Valombrose, 06189 Nice Cedex 2, France. ${ }^{11}$ Group of Nutrition and Dietary Committees of Anti-Cancer Centers (Interclan CLCC), Villejuif, France. ${ }^{12}$ Agricultural, Food and Nutritional Science, University of Alberta, Edmonton, AB T6G 2P5, Canada.

\section{Authors' contributions}

EGN was involved in data analysis, data interpretation and manuscript writing, RDK was involved in drafting the manuscript and revising it critically, CJ was involved in data collection at the Cancer Institute of Montpellier and manuscript preparation, MJ was involved in data analysis and manuscript preparation, JM was involved in data collection at the Curie Institute of Paris, BP was involved in data collection at Centre Francois Baclesse of Caen, SB was involved in data collection at the Centre Antoine Lacassagne of Nice, GN was involved in revising the manuscript critically, JC was involved in manuscript draft, WW was involved in data interpretation, in particular the intercultural angles, and manuscript preparation, ST was involved in data analysis and manuscript preparation, and PS was involved in conceiving the study, study design, data interpretation and manuscript preparation. All authors read and approved the final manuscript.

\section{Acknowledgements}

This study was supported by the SIRIC Montpellier Cancer (Grant INCa-DGOSInserm 6045) which was not involved in study design; in the collection, analysis and interpretation of data; in the writing of the report; and in the decision to submit the article for publication. All authors critically reviewed the manuscript and approved the version for submission. The authors are grateful to the Curie Institute of Paris, the Centre Antoine Lacassagne of Nice, the Centre Francois Baclesse of Caen, and the Paoli Calmettes Institute of Marseille, in particular Françoise Ducès, for taking part in the study and for supplying the completed questionnaires. They would like to thank Eliane Courties for advice and expertise in the dietary industry, and Pauline Monier for assistance in filling in the questionnaires. They also thank Pr. Marc Ychou and Dr. Vanessa Guillaumon for their support. They are grateful to the dietetic staff for their assistance with patient recruitment toward the end of the study and their support in completing the study.

\section{Competing interests}

The authors declare that they have no competing interests.

Received: 21 January 2016 Accepted: 8 June 2016

Published online: 21 June 2016

\section{References}

Andreyev HJN, Norman AR, Oates J, Cunningham D (1998) Why do patients with weight loss have a worse outcome when undergoing chemotherapy for gastrointestinal malignancies? Eur J Cancer 34:503-509. doi:10.1016/S0959-8049(97)10090-9

Arends J, Bodoky G, Bozzetti F et al (2006) ESPEN guidelines on enteral nutrition: non-surgical oncology. Clin Nutr Edinb Scotl 25:245-259. doi:10.1016/j.clnu.2006.01.020

Argilés JM (2005) Cancer-associated malnutrition. Eur J Oncol Nurs 9:S39-S50. doi:10.1016/j.ejon.2005.09.006

August DA, Huhmann MB, American Society for Parenteral and Enteral Nutrition (A.S.P.E.N.) Board of Directors (2009) A.S.P.E.N. clinical guidelines: nutrition support therapy during adult anticancer treatment and in hematopoietic cell transplantation. JPEN J Parenter Enteral Nutr 33:472-500. doi:10.1177/0148607109341804

Auvray M, Spence C (2008) The multisensory perception of flavor. Conscious Cogn 17:1016-1031. doi:10.1016/j.concog.2007.06.005

Bauer J (2007) Nutritional management and dietary guidelines for cancer cachexia. Eur Oncol Haematol 00:12. doi:10.17925/EOH.2007.0.2.12

Bellisle F (2003) Contribution of snacks and meals in the diet of French adults: a diet-diary study. Physiol Amp Behav 79:183-189. doi:10.1016/ S0031-9384(03)00088-X

Bellisle F, Dalix AM, Mennen L et al (2003) Contribution of snacks and meals in the diet of French adults: a diet-diary study. Physiol Behav 79:183-189. doi:10.1016/S0031-9384(03)00088-X

Boltong A, Keast R (2012) The influence of chemotherapy on taste perception and food hedonics: a systematic review. Cancer Treat Rev 38:152-163. doi:10.1016/j.ctrv.2011.04.008

Boltong A, Keast R, Aranda S (2012) Experiences and consequences of altered taste, flavour and food hedonics during chemotherapy treatment. Support Care Cancer 20:2765-2774. doi:10.1007/s00520-012-1398-7

Bortolon C, Krikorian A, Carayol M et al (2014) Cancer-related fatigue in breast cancer patients after surgery: a multicomponent model using partial least squares-path modeling: a multicomponent model of fatigue in breast cancer. Psychooncology 23:444-451. doi:10.1002/pon.3438

Brisbois TD, de Kock IH, Watanabe SM et al (2011) Characterization of chemosensory alterations in advanced cancer reveals specific chemosensory phenotypes impacting dietary intake and quality of life. J Pain Symptom Manag 41:673-683. doi:10.1016/j.jpainsymman.2010.06.022

Coa Kl, Epstein JB, Ettinger D et al (2015) The impact of cancer treatment on the diets and food preferences of patients receiving outpatient treatment. Nutr Cancer 67:339-353. doi:10.1080/01635581.2015.990577

de Castro J (2000) Palatability and intake relationships in free-living humans characterization and independence of influence in North Americans. Physiol Behav 70:343-350. doi:10.1016/S0031-9384(00)00264-X

de Castro JM, Bellisle F, Dalix AM (2000) Palatability and intake relationships in free-living humans: measurement and characterization in the French. Physiol Behav 68:271-277. doi:10.1016/50031-9384(99)00166-3

Dewys WD, Begg C, Lavin PT et al (1980) Prognostic effect of weight loss prior to chemotherapy in cancer patients. Eastern Cooperative Oncology Group. Am J Med 69:491-497

Epstein JB, Barasch A (2010) Taste disorders in cancer patients: pathogenesis, and approach to assessment and management. Oral Oncol 46:77-81. doi:10.1016/j.oraloncology.2009.11.008

Epstein JB, Phillips N, Parry J et al (2002) Quality of life, taste, olfactory and oral function following high-dose chemotherapy and allogeneic hematopoietic cell transplantation. Bone Marrow Transpl 30:785-792. doi:10.1038/ sj.bmt. 1703716

Fearon K, Strasser F, Anker SD et al (2011) Definition and classification of cancer cachexia: an international consensus. Lancet Oncol 12:489-495. doi:10.1016/S1470-2045(10)70218-7

Gamper E-M, Zabernigg A, Wintner LM et al (2012) Coming to your senses: detecting taste and smell alterations in chemotherapy patients. A systematic review. J Pain Symptom Manag 44:880-895. doi:10.1016/j. jpainsymman.2011.11.011

Hovan AJ, Williams PM, Stevenson-Moore P et al (2010) A systematic review of dysgeusia induced by cancer therapies. Support Care Cancer 18:10811087. doi:10.1007/s00520-010-0902-1 
Hutton JL, Baracos VE, Wismer WV (2007) Chemosensory dysfunction is a primary factor in the evolution of declining nutritional status and quality of life in patients with advanced cancer. J Pain Symptom Manag 33:156-165. doi:10.1016/j.jpainsymman.2006.07.017

Irune E, Dwivedi RC, Nutting CM, Harrington KJ (2014) Treatment-related dysgeusia in head and neck cancer patients. Cancer Treat Rev 40:1106-1117. doi:10.1016/j.ctrv.2014.06.011

Isenring EA, Bauer JD, Capra S (2007) Nutrition support using the American Dietetic Association medical nutrition therapy protocol for radiation oncology patients improves dietary intake compared with standard practice. J Am Diet Assoc 107:404-412. doi:10.1016/j.jada.2006.12.007

Isenring E, Capra S, Bauer J (2012) Nutrition support, quality of life and clinical outcomes. J Hum Nutr Diet 25:505-506; author reply 507-508. doi:10.1111/j.1365-277X.2011.01221.x

Kong H, Kwon OK, Yu W (2012) Changes of quality of life after gastric cancer surgery. J Gastric Cancer 12:194-200. doi:10.5230/jgc.2012.12.3.194

Laurienti PJ, Burdette JH, Maldjian JA, Wallace MT (2006) Enhanced multisensory integration in older adults. Neurobiol Aging 27:1155-1163. doi:10.1016/j.neurobiolaging.2005.05.024

McQuestion M, Fitch M, Howell D (2011) The changed meaning of food: physical, social and emotional loss for patients having received radiation treatment for head and neck cancer. Eur J Oncol Nurs Off J Eur Oncol Nurs Soc 15:145-151. doi:10.1016/j.ejon.2010.07.006

Meuric J, Besnard I (2012) Nutrition chez le patient adulte atteint de cancer: quand doit-on proposer un conseil diététique personnalisé? Nutr Clin Métab 26:197-218. doi:10.1016/j.nupar.2012.10.006

Minton O, Berger A, Barsevick A et al (2013) Cancer-related fatigue and its impact on functioning: cancer-related fatigue. Cancer 119:2124-2130. doi:10.1002/cncr.28058

Okkels SL, Bredie WLP, Klausen TW, Beck AM (2016) An investigation into between-meal food desires among hospitalised haematological cancer patients. Clin Nutr Edinb Scotl 35:440-445. doi:10.1016/j.clnu.2015.03.010

Ravasco P, Monteiro-Grillo I, Marques Vidal P, Camilo ME (2005a) Impact of nutrition on outcome: a prospective randomized controlled trial in patients with head and neck cancer undergoing radiotherapy. Head Neck 27:659-668. doi:10.1002/hed.20221
Ravasco P, Monteiro-Grillo I, Vidal PM, Camilo ME (2005b) Dietary counseling improves patient outcomes: a prospective, randomized, controlled trial in colorectal cancer patients undergoing radiotherapy. J Clin Oncol Off J Am Soc Clin Oncol 23:1431-1438. doi:10.1200/JCO.2005.02.054

Ruo Redda MG, Allis S (2006) Radiotherapy-induced taste impairment. Cancer Treat Rev 32:541-547. doi:10.1016/j.ctrv.2006.06.003

Sandelowski M (2010) What's in a name? Qualitative description revisited. Res Nurs Health 33:77-84

Senesse P, Bachmann P, Bensadoun R-J et al (2014) Clinical nutrition guidelines of the French Speaking Society of Clinical Nutrition and Metabolism (SFNEP): summary of recommendations for adults undergoing nonsurgical anticancer treatment. Dig Liver Dis 46:667-674. doi:10.1016/j. dld.2014.01.160

Servaes P, Verhagen S, Bleijenberg G (2002) Determinants of chronic fatigue in disease-free breast cancer patients: a cross-sectional study. Ann Oncol 13:589-598

Small DM (2012) Flavor is in the brain. Physiol Behav 107:540-552. doi:10.1016/j.physbeh.2012.04.011

Spence C (2013) Multisensory flavour perception. Curr Biol 23:R365-R369. doi:10.1016/j.cub.2013.01.028

Stevenson R (2009) The psychology of flavour. Oxford University Press, Oxford

Thibault R, Chikhi M, Clerc A et al (2011) Assessment of food intake in hospitalised patients: a 10-year comparative study of a prospective hospital survey. Clin Nutr 30:289-296. doi:10.1016/j.clnu.2010.10.002

Tomlinson D, Hinds PS, Ethier M-C et al (2013) Psychometric properties of instruments used to measure fatigue in children and adolescents with cancer: a systematic review. J Pain Symptom Manage 45:83-91. doi:10.1016/j.jpainsymman.2012.02.010

Verhagen JV (2007) The neurocognitive bases of human multimodal food perception: consciousness. Brain Res Rev 53:271-286. doi:10.1016/j. brainresrev.2006.09.002

Zabernigg A, Gamper E-M, Giesinger JM et al (2010) Taste alterations in cancer patients receiving chemotherapy: a neglected side effect? Oncologist 15:913-920. doi:10.1634/theoncologist.2009-0333

\section{Submit your manuscript to a SpringerOpen ${ }^{\circ}$ journal and benefit from:}

- Convenient online submission

- Rigorous peer review

- Immediate publication on acceptance

- Open access: articles freely available online

- High visibility within the field

- Retaining the copyright to your article

Submit your next manuscript at springeropen.com 